\title{
Survey on Medical Imaging of Electrical Impedance Tomography (EIT) by Variable Current Pattern Methods
}

\author{
Dr. Edriss Eisa Babikir Adam, \\ Assistant Professor / EEE, \\ Mainefhi College of Engineering and Technology, \\ Mainefhi, Eritrea. \\ bonzoga20@gmail.com
}

Prof. Sathesh,

Department of EEE,

Eritrea Institute of Technology,

Eritrea.

sathesh4you@gmail.com

\begin{abstract}
Recently, the image reconstruction study on EIT plays a vital role in the medical application field for validation and calibration purpose. This research article analyzes the different types of reconstruction algorithms of EIT in medical imaging applications. Besides, it reviews many methods involved in constructing the electrical impedance tomography. The spatial distribution and resolution with different sensitivity has been discussed here. The electrode arrangement of various methods involved in the EIT system is discussed here. This research article comprises of adjacent drive method, cross method, and alternative opposite current direction method based on the voltage driven pattern. The assessment process of biomedical EIT has been discussed and investigated through the impedance imaging of the existent substances. The locality of the electrodes can be calculated and fixed for appropriate methods. More specifically, this research article discusses about the EIT image reconstruction methods and the significance of the alternative opposite current direction approach in the biomedical system. The change in conductivity test is further investigated based on the injection of current flow in the system. It has been established by the use of Electrical Impedance
\end{abstract}


Tomography and Diffuse Optical Tomography Reconstruction Software (EDITORS) software, which is open-source software.

Keywords: Electrical Impedance Tomography, Medical devices

\section{INTRODUCTION}

Non-invasive modality imaging is generally utilized in biomedical field for image conductivity subjects. It is based on many methods such as impedance and resistance, which is called as electrical impedance tomography and electrical resistance tomography respectively [1]. These electrical measurements are obtained from various electrodes, which are attached to the boundary of the internal conductivity distribution subject [2]. Generally, the electrodes are fixed on the surface of the subject and it is receives current from the injection process involved in EIT. The stimulation for current is varied according to the amplitude and frequency in the range of 1 to $100 \mathrm{kHz}$. The potential drop can be measured by using some voltage measuring electrodes [3]. The image reconstruction methods calculate local conductivity distribution from the border data. EIT can be used in many fault detection applications of the medical imaging domain [4]. The lung problems in medical sectors can be solved by EIT, which monitors the heart function, blood flow, and collapsed lung function. The premenstrual syndrome can be determined from the intracellular versus extracellular fluid [5]. The workflow of EIT system is a combination of frequency generator, phase shifter, multiplier, demodulator, and signal conditioner that are connected with objects via multiplexers and several electrodes [6]. The simplified structure of the EIT system [29] is shown in figure 1. 


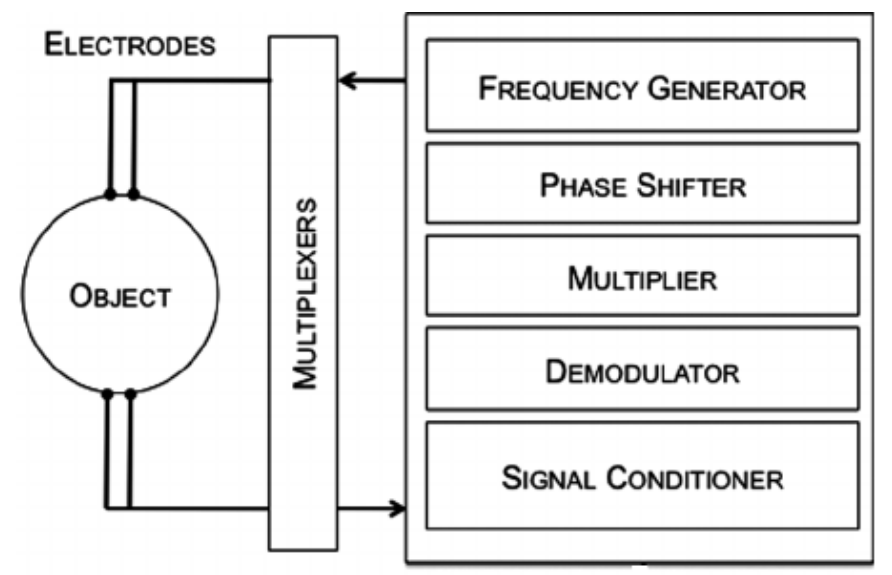

Figure 1 Block diagram of EIT system work flow [29]

This system is attached to the surface of the object by using the collection of electrodes. The generator is used to provide power to the system [7]. The data acquisition system has been incorporated with current injector and different data channels to receive the information from the object [8]. The voltage measurement details can be extracted from the demodulator section, which remains as the most essential part of the device. The signal conditioner circuit is used for many purposes such as noise filtering and signal converting processes [9]. Finally, it is responsible for interfacing a computer. The voltage measurement data of objects are captured and the images are reconstructed with the help of an image reconstruction algorithm, which is already incorporated in the EIT system [10].

The temperature changes for many treatments can be measured for avoiding harm in medical sectors. The improving electrocardiogram (ECG) is associated with the measured internal conductivity distributions [11]. Impedance distributions are structured with the properties of the objects based on several bioelectrical properties of the tissues in medical applications. The injected current flow can be directed by the impedance within the boundary of the object [12]. The big changes in impedance level of the object with respect to various voltage changes at the surface are called ill-posed problems in EIT [13]. Generally, this EIT system is very cost efficient and user friendly. 


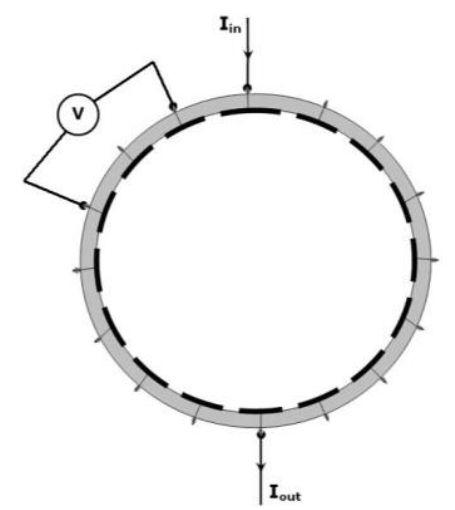

Figure 2 Adjacent Electrode Collections [30]

Figure 2 shows the setup of adjacent electrode collections [30]. Recently, many methods are proposed to reduce the hazards detected in stimulation of the human body during x-ray exposure. The multi-specialty hospitals include a continuous monitoring system for any patients near the bedside [14] [15] [16]. The data acquisition hardware is an essential part of the EIT system, which will acquire the data from the objects. Nowadays, the image reconstruction software will be incorporated with the EIT system, which remains as the essential part of the device [17].

\section{ORGANIZATION OF THE RESEARCH}

This research article comprises several sections as follows; section 3 discusses about the related works on EIT. Section 4 proposes the methodologies for medical applications. Section 5 examines and discusses several testing for EIT. Future tasks and enhancement are discussed in section 6 .

\section{PRELIMINARIES}

Many research articles are focused on simulation and measurement in the field of tomography for various methods such as trigonometric measurement-based methods. EIT can be reconstructed based on voltage and current on an object with the conditions of permittivity and conductivity. Generally, the current will shoot up from the electrodes due to its contact with the surface. This procedure can be used as conditions of the current to find the voltage [18]. Based 
on that, the current injecting electrodes are used to determine the voltage patterns. Besides, in many applications, the voltage measuring electrodes and current patterns are used for measurement purpose [19].

Gisser et al have introduced an adaptive method for the current injection method in EIT. They investigate various current injecting methods. This method is also called as a trigonometric method due to its structure. Here, the current is injected into all electrodes for obtaining the measurement between current and voltage. Every pair of electrodes provides a potential drop between those points [20]. Besides they insist that, holding many current injectors are required to inject current into the electrodes. The number of electrode EIT systems is directly proportional to the number of current injectors [21]. The boundary potential in every measurement depends on the electrodes. As an adaptive method, they used 16 electrode EIT systems to produce voltages based on the current flow. This flow creates a potential difference to measure the voltage between the electrodes. The main limitation of these approaches is the need for the driver support to inject current [22].

The polar-driven method is used in many research articles for the EIT system. Commonly, it is used in brain tomography-type machines or systems. The voltage reference electrodes provide a potential difference from the current injecting electrodes [23] [24]. The serially connected electrodes are carrying a current flow, which develops a potential difference between adjacent electrodes and differential voltage data. The potential difference can be measured between all pairs of electrodes after current injection [25]. This method includes 208 voltage measurements with a 16 electrode EIT system. It can produce a maximum of 13 voltage differential data. This method can produce less current injection, when compared to the adjacent method and approach. But it is insensitive and distribution with greater uniform distribution property for the body image. The conductivity at the boundary level cannot be predicted early; therefore less sensitive Priori boundary distribution has been obtained[26].

\section{METHODOLOGIES}

\subsection{Adjacent Drive Method}


The other name of this method is called a neighboring method, which is used for the current-driven pattern. The adjacent electrodes are carrying current and voltage and it can be measured between adjacent probes of electrode pairs. The 16 electrodes are available in the EIT system for developing the circular structure between electrodes 1 to 16 . Generally, the current is injected first at electrode 1 sequentially to measure the voltages accordingly [27]. Due to the current projection calculation, the voltages between some special paths in the current cannot be measured. Their electrode pairs are 16 to 1, 1-2, and 2-3. Therefore, the 13 differential voltage data can be acquired from the EIT system.

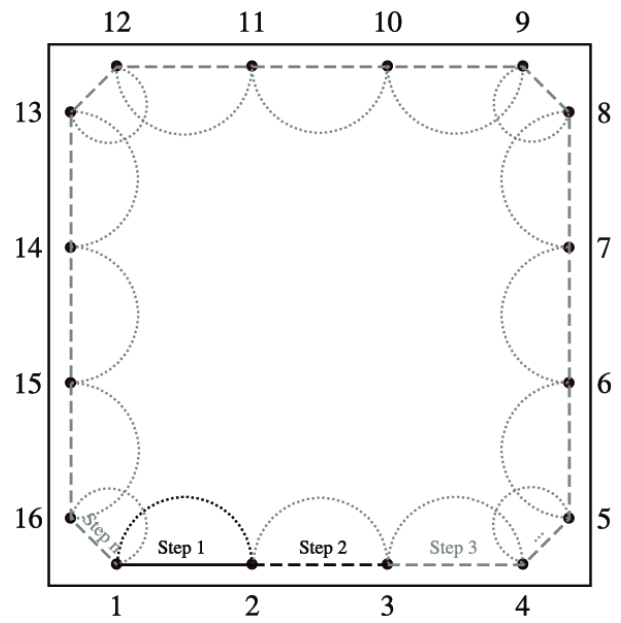

Figure 3 Adjacent Drive Method Pattern [31]

Figure 3 shows the procedure of adjacent drive pattern [31]. The different electrodes can be used as initial electrodes to inject the current. The data frame will be used in adjacent drive methods. This provides relief from unknown contact impedance problems to measure the voltages obtained from the current flow [28]. The current injected electrodes have reduced the number of measurements by using the formula $\mathrm{N}(\mathrm{N}-3)$. The measurements are independent to set the mutual impedance during the injection on measurement pairs.

\subsection{Cross Method}

The diagonal pattern approach process can be called as cross method. This method selects current and voltage references sequentially. This method is used in a very rare phenomenon. The 
current is injected through the $16^{\text {th }}$ electrode is taken as the reference of the $1^{\text {st }}$ electrode. But the current is injecting two electrodes only, which are titled as the $16^{\text {th }}$ electrode and $2^{\text {nd }}$ electrode. The electrode pairs can be constructed to measure the voltage reference by using the current flow. But here, it can be made as 91 measurements due to the utilization of limited electrodes for measuring the voltage references [15]. Figure 4 has shown the current-driven pattern like a cross structure [32].

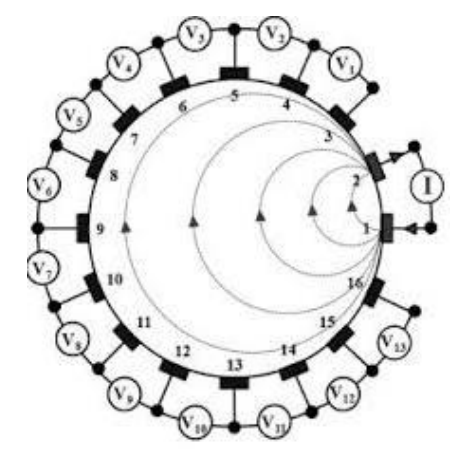

Figure 4 Cross current driven pattern [32]

\subsection{Proposed alternative opposite current driven pattern Method}

The recovering problem can be solved through unknown conductivity obtained from the border information. This problem is addressed as an ill-posed problem and a solution can be obtained through impedance variation observed in the current pattern. In all other methods, electric current flow is nonlinear due to the non-uniformity volume of the conductor. These are the fundamental problems in image reconstruction. The applied currents are flowing through the volume conductor with a source to measure the potential drop. The mathematical model has been proposed with the Laplace equation and it is defined as,

$$
\nabla \cdot \rho^{-1}(\nabla \varnothing)=0, \quad x, y \in D
$$

Where, $\rho$ is resistive distribution, $\varnothing$ is voltage information, and D is examined field.

The voltage is predicted with the following current equation on the examining field $\mathrm{D}$ and it considers constant resistivity. The actual resistivity distribution is derived from the surface measurement with inverse circulation. 


\section{Remarks:}

$$
\emptyset_{\mid \partial D}=V_{0}(x, y)
$$

$\rho^{-1} \frac{\partial y}{\partial x \mid \partial D}=J_{0}$

Where, $V_{0}$ is the measuring voltage at optimum conditions, $J_{0}$ is the current density on boundary, $\partial D$ is the boundary of $\mathrm{D}$.

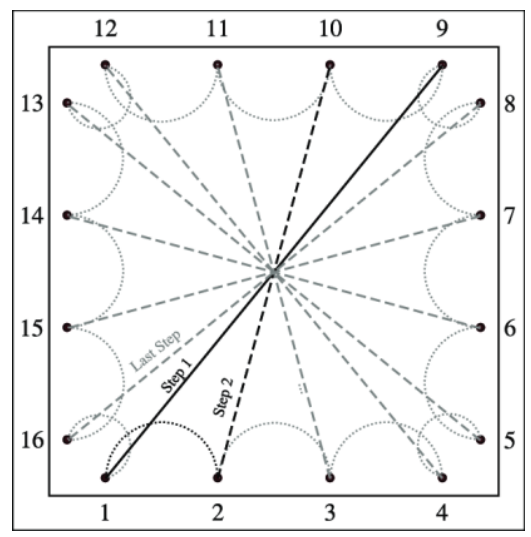

Figure 5 Alternative Opposite Current Driven Pattern [31]

Figure 5 shows the alternative opposite current driven pattern [31]. Different methods are incorporated to solve the forward problem. The finite difference method (FDM), finite element method (FEM) and Boundary element method (BEM) are available to solve the forward problem in the EIT system. The ill-posed problem of EIT can be solved by using regularization techniques. Table 1 shows the different problems in EIT system during the procedure.

Table 1 Addressing Following Problems by Alternative Opposite Current

\begin{tabular}{|c|c|}
\hline Forward problem & Inverse Problem \\
\hline Model parameter to Data & Data to Model Parameter \\
\hline Well-Posed & Ill-Posed and nonlinear \\
\hline
\end{tabular}


Besides, ill-posed problems require smooth assumptions and prior information to solve the inverse problem. This type of regularization can provide a good impact during the medical imaging at scan, which can be minimize the over-fitting problem. The unexpected random conditions have calculated the reasonable solutions. This method can be categorized into three types, as follows;

1. Back projection Method

2. Iterative Method

3. One-step Linearized Method

\section{RESULTS \& DISCUSSION}

Figure 6 shows step 1 to step 16 by acquiring the voltage information [31]. The images are reconstructed from the boundary information obtained from the phantom with the help of adjacent electrodes. EIT has been reconstructed with EDITORS software, which is open-source software. The proposed model has reconstructed the images from diffuse optical information. The conductivity can be changed based on the FEM method. The forward problem can be solved by using the FEM. The creation of reconstruction images is done by EDITORS software for the review.
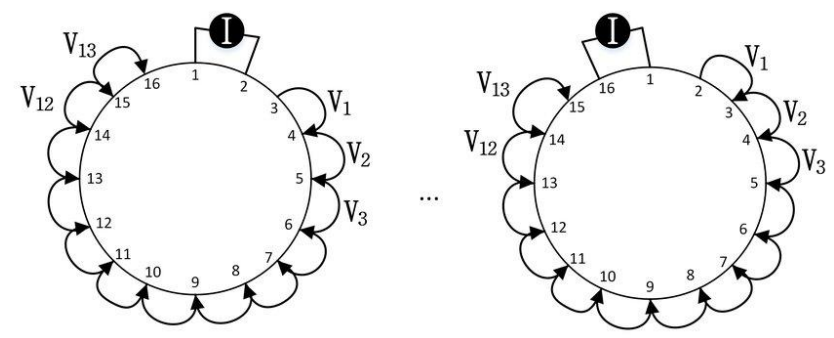

Figure 6 Step by Step Examination Procedure [33]

The voltage pattern of various stimulation structures can be measured based on the injected current. The potential differences are computed by using the sequence electrodes. Here, the forward problems are determined by utilizing FEM models for further process. Figure 7 shows different voltage patterns for adjacent stimulation by injecting the current pattern. 

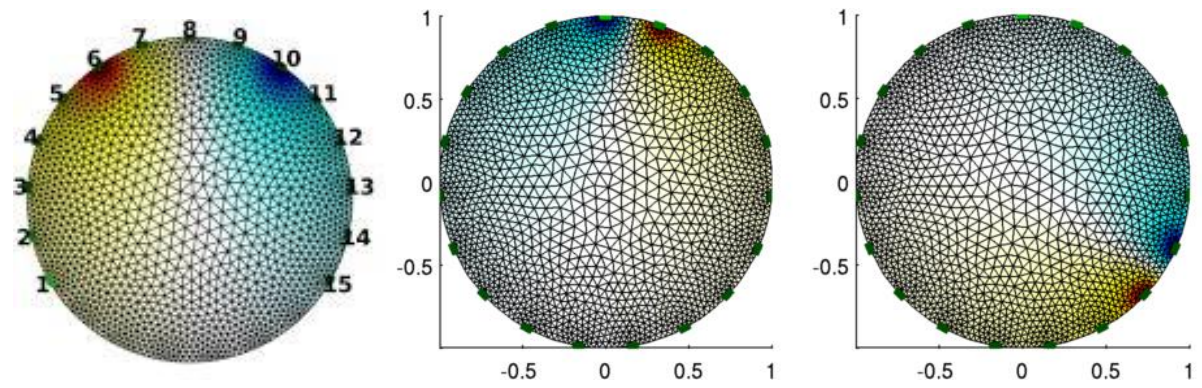

Figure 7 Different Voltage Patterns for Adjacent Stimulation [34]

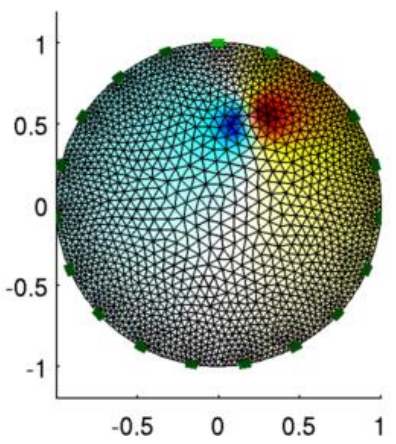

Figure 8 Voltage Pattern Change in Conductivity [34]

The EIT images are used to compute the air circulation associated with X-Ray imaging for identifying the ventilated lung region. This morphological structure of the lung can be reflected in the gas exchange regional information. EIT images can display the region of the lung sections in the thoracic images. The change in conductivity of the voltage pattern can resolve the non-linearity process. Figure 8 shows the change in conductivity of the voltage pattern.

\section{CONCLUSION}

Thus, EIT system is used to assess any region with a great potential for new medical imaging. This EIT imaging modality is radiation-free, portable and inexpensive. Many methods are available to assess the regional medical images with tidal volume and determined variation in the objects. These proposed EIT system methods are capable to reach higher rates in measurement and reconstruction algorithms, which prove the robustness in sensing the unit 
errors. Besides, it reduces the image artifacts, which are used to spread over the noise in the system. There is a good management procedure in acute and weaning points of the moving exposure to the air. Finally, this EIT system enables the following conditions such as the affordable cost; images should be received in standard formats. Also, the system is robust against probe touch problems with noise complications. The clinical users are expecting more robust and sophisticated real-time output with more clarity on low-dose radiation. This procedure should be further improved in the future work. EIT is very useful in the diagnosis sector of pulmonary problems but there is the absence of compactness in the existing system. To immediately enhance the clinical decision-making process with higher accuracy is suitable for diagnosing the pulmonary problems around the bedside. The procedure can be extended as future work. The reconstruction software will modify the spontaneous interface involved in a real-time medical imaging domain.

\section{REFERENCES}

[1] D. Liu, D. Gu, D. Smyl, J. Deng, and J. Du, "B-spline-based sharp feature preserving shape reconstruction approach for electrical impedance tomography," IEEE Trans. Med. Imag., vol. 38, no. 11, pp. 2533-2544, Nov. 2019.

[2] M. Lu, Y. Xie, W. Zhu, A. Peyton, and W. Yin, "Determination of the magnetic permeability, electrical conductivity, and thickness of ferrite metallic plates using a multifrequency electromagnetic sensing system," IEEE Trans. Ind. Informat., vol. 15, no. 7, pp. 4111-4119, Jul. 2019.

[3] D. Liu, D. Gu, D. Smyl, J. Deng, and J. Du, "B-Spline-based sharp feature preserving shape reconstruction approach for electrical impedance tomography," IEEE Trans. Med. Imag., vol. 38, no. 11, pp. 2533-2544, Nov. 2019.

[4] S. J. Ren, M. Soleimani, Y. Y. Xu, and F. Dong, "Inclusion boundary reconstruction and sensitivity analysis in electrical impedance tomography," Inverse Probl. Sci. En., vol. 26, no. 7, pp. 1037-1061, 2018. 
[5] E. Beretta, S. Micheletti, S. Perotto, and M. Santacesaria, "Reconstruction of a piecewise constant conductivity on a polygonal partition via shape optimization in EIT," J. Comput. Phys., vol. 353, pp. 264-280, 2018.

[6] K. Krishnan, J. Liu, and K. Kohli, "Feature-space assessment of electrical impedance tomography coregistered with computed tomography in detecting multiple contrast targets," Med. Phys., vol. 41, no. 6, pp. 061903, 2014.

[7] B. Schullcke, B. Gong, S. Krueger-Ziolek, M. Soleimani, U. Mueller- Lisse, and K. Moeller, "Structural-functional lung imaging using a combinedCT- EIT and a discrete cosine transformation reconstructionmethod," Sci. Rep-uk., vol. 6, no. 1, pp. 25951, 2016.

[8] S. Teniou and M. Meribout, "A multimodal image reconstruction method using ultrasonic waves and electrical resistance tomography," IEEE Trans.Image Process., vol. 24, no. 11, pp. 3512-3521, Nov. 2015.

[9] G. H. Liang, S. J. Ren, S. Zhao, and F. Dong, "A Lagrange-Newtonmethod for EIT/UT dualmodality image reconstruction," Sensors, vol. 19, no. 9, pp. 1966, 2019.

[10] D. Ackermann and G. Schmitz, "Detection and tracking of multiple microbubbles in ultrasound B-mode images," IEEE Trans. Ultrason., Ferroelect. Freq. Control., vol. 63, no. 1, pp. 72-82, Jan. 2015.

[11] N. Duric et al., "Using whole breast ultrasound tomography to improve breast cancer risk assessment: A novel risk factor based on the quantitative tissue property of sound speed," J. Clin. Med., vol. 9, no. 2, pp. 367(12), 2020.

[12] A. F. Firdaus and M. Meribout, "A new parallel VLSI architecture for real-time electrical capacitance tomography," IEEE Trans. Comput., vol. 65, no. 1, pp. 30-41, Jan. 2016.

[13] L. Wen, C. Tan, F. Dong, and S. Zhao, "Design of ultrasonic tomography system for biomedical imaging," in Proc. IEEE I2MTC, Auckland, New Zealand, May 2019, pp. 1-5.

[14] D. Liu, D. P. Gu, D. Smyl, J. S. Deng, and J. F. Du, "B-spline based sharp feature preserving shape reconstruction approach for electrical impedance tomography," IEEE Trans. Med. Imag., vol. 14, no. 8, pp. 1-12, Aug. 2019. 
[15] S. Liu, H. Wu, Y. Huang, Y. Yang, and J. Jia, "Accelerated structure-aware sparse Bayesian learning for three-dimensional electrical impedance tomography," IEEE Trans. Ind. Informat., vol. 15, no. 9, pp. 5033-5041, Sep. 2019.

[16] F. Dong, Y. Xu, L. Hua, and H.Wang, "Two methods for measurement of gas-liquid flows in vertical upward pipe using dual-plane ERT system," IEEE Trans. Instrum. Meas., vol. 55, no. 5, pp. 1576-1586, Oct. 2006.

[17] M. Meribout and I. M. Saied, "Real-time two-dimensional imaging of solid contaminants in gas pipelines using an electrical capacitance tomography system," IEEE Trans. Ind. Electron., vol. 64, no. 5, pp. 3989-3996, May 2017.

[18] J. Sun et al., "Proportional-integral controller modified landweber iterative method for image reconstruction in electrical capacitance tomography," IEEE Sensors J., vol. 19, no. 19, pp. 8790-8802, Oct. 2019.

[19] M. Cheney, D. Isaacson, and J. C. Newell, "Electrical impedance tomography," SIAM Rev., vol. 41, no. 1, pp. 85-101, 1999.

[20] Gisser G, Isaacson D, Newell JC, "Current topics in impedance imaging”, Clin Physiol Meas, 1987, 8:38-46.

[21] Y. Yang, H. Wu, and J. Jia, "Image reconstruction for electrical impedance tomography using enhanced adaptive group sparsity with total variation," IEEE Sensors J., vol. 17, no. 17, pp. 5589-5598, Sep. 2017.

[22] D. Liu, D. Smyl, and J. Du, "A parametric level set-based approach to difference imaging in electrical impedance tomography," IEEE Trans. Med. Imag., vol. 38, no. 1, pp. 145-155, Jan. 2019.

[23] M. Wang, Industrial Tomography: Systems and Applications, M. Wang, ed, 1st ed. Cambridge, U.K.: Woodhead, 2015. ch. 2.

[24] M. Arad, S. Zlochiver, T. Davidson, Y. Shoenfeld, A. Adunsky, and S. Abboud, "The detection of pleural effusion using a parametric EIT technique," Physiol. Meas., vol. 30, no. 4, pp. 421-428, Apr. 2009. 
[25] C. Gomez-Laberge, J. H. Arnold, and G. K. Wolf, "A unified approach for EIT imaging of regional overdistension and atelectasis in acute lung injury," IEEE Trans. Med. Imag., vol. 31, no. 3, pp. 834-842, Mar. 2012.

[26] K. Y. Aristovich, B. C. Packham, H. Koo, G. S. D. Santos, A. McEvoy, and D. S. Holder, "Imaging fast electrical activity in the brain with electrical impedance tomography," NeuroImage, vol. 124, pp. 204-213, Jan. 2016.

[27] M. Cheney, D. Isaacson, J. C. Newell, S. Simske, and J. Goble, "NOSER: An algorithm for solving the inverse conductivity problem," Int. J. Imag. Syst. Technol., vol. 2, no. 2, pp. 66-75, 1990.

[28] W. Q. Yang and L. Peng, "Image reconstruction algorithms for electrical capacitance tomography," Meas. Sci. Technol., vol. 14, no. 1, pp. R1-R13, Jan. 2003.

[29] Faia, P. M., R. Silva, M. G. Rasteiro, F. A. P. Garcia, A. R. Ferreira, M. J. Santos, J. B. Santos, and A. P. Coimbra. "Imaging particulate two-phase flow in liquid suspensions with electric impedance tomography." Particulate Science and Technology 30, no. 4 (2012): 329-342. [30] Liu, Hong. n.d. "EIT Sensors - Sensor for Electrical Impedance Tomography - University of Leeds." Leeds.Ac.Uk. https://www.leeds.ac.uk/olil/tomography/Sensors.php.

[31] Haingartner, Mathias, Sandra Gschoßmann, Max Cichocki, and Martin Schagerl. "Improved current injection pattern for the detection of delaminations in carbon fiber reinforced polymer plates using electrical impedance tomography." Structural Health Monitoring (2020): 1475921720972308 .

[32] Harikumar, R., R. Prabu, and S. Raghavan. "Electrical impedance tomography (EIT) and its medical applications: a review." Int. J. Soft Comput. Eng 3, no. 4 (2013): 193-198.

[33] Russo, Stefania, Samia Nefti-Meziani, Nicola Carbonaro, and Alessandro Tognetti. "Development of a high-speed current injection and voltage measurement system for electrical impedance tomography-based stretchable sensors." Technologies 5, no. 3 (2017): 48.

[34] http://eidors3d.sourceforge.net/tutorial/EIDORS_basics/forward_solvers.shtml. "EIDORS." n.d. Sourceforge.Net. 